\title{
Control of Glyphosate-Resistant Common Waterhemp (Amaranthus rudis) in Glufosinate-Resistant Soybean
}

\author{
Amit J. Jhala, Lowell D. Sandell, Debalin Sarangi, Greg R. Kruger, and Steven Z. Knezevic*
}

Glyphosate-resistant (GR) common waterhemp has become a significant problem weed in Nebraska and several Midwestern states. Several populations of GR common waterhemp are also resistant to acetolactate synthase (ALS)-inhibiting herbicides, making them difficult to control with POST herbicides in GR soybean. Glufosinate-resistant (GFR) soybean is an alternate system for controlling GR common waterhemp, justifying the need for evaluating glufosinate-based herbicide programs. The objectives of this study were to compare POST-only herbicide programs (including one-pass and two-pass POST programs) with PRE followed by (fb) POST herbicide programs for control of GR common waterhemp in GFR soybean and their effect on common waterhemp density, biomass, and soybean yield. Field experiments were conducted in 2013 and 2014 near Fremont, NE in a grower's field infested with GR common waterhemp. Glufosinate applied early- and late-POST provided 76\% control of GR common waterhemp at $14 \mathrm{~d}$ after late-POST (DALPOST) compared with 93\% control with a PRE fb POST program when averaged across treatments. The PRE application of chlorimuron plus thifensulfuron plus flumioxazin, $S$-metolachlor plus fomesafen or metribuzin, saflufenacil plus dimethenamid-P fb glufosinate provided $\geq 95 \%$ control of common waterhemp throughout the growing season, reduced common waterhemp density to $\leq 2.0$ plants $\mathrm{m}^{-2}$, caused $\geq 94 \%$ biomass reduction, and led to 1,984 to $2,210 \mathrm{~kg} \mathrm{ha}^{-1}$ soybean yield. Averaged across treatments, the PRE $\mathrm{fb}$ POST program provided $82 \%$ common waterhemp control at soybean harvest, reduced density to 23 plants $\mathrm{m}^{-2}$ at 14 DALPOST, and caused $86 \%$ biomass reduction and $1,803 \mathrm{~kg} \mathrm{ha}^{-1}$ soybean yield compared with $77 \%$ control, 99 plants $\mathrm{m}^{-2}, 53 \%$ biomass reduction, and $1,190 \mathrm{~kg} \mathrm{ha}^{-1}$ yield with POST-only program. It is concluded that PRE fb POST programs with multiple effective modes of action are available for control of GR common waterhemp in GFR soybean.

Nomenclature: Acetochlor, alachlor, cloransulam, chlorimuron, dimethenamid, flumioxazin, fomesafen, glufosinate, glyphosate, imazethapyr, metribuzin, saflufenacil, $S$-metolachlor, sulfentrazone, thifensulfuron, common waterhemp, Amaranthus rudis Sauer, soybean, Glycine max (L.) Merr.

Key words: Biomass reduction, POST-only program, PRE followed by POST, resistance management, soybean yield.

\begin{abstract}
Amaranthus rudis resistente a glyphosate (GR) se ha convertido en un problema de malezas significativo en Nebraska y en varios estados del Medio Oeste. Varias poblaciones de $A$. rudis GR también son resistentes a herbicidas inhibidores de acetolactate synthase, lo que las hace difíciles de controlar con herbicidas POST en soja GR. Soja resistente a glufosinate es un sistema alternativo para el control de $A$. rudis GR, lo que justifica la necesidad de evaluar programas de herbicidas basados en glufosinate. Los objetivos de este estudio fueron comparar programas con sólo herbicidas POST (incluyendo programas POST con uno y dos pases) con programas de herbicidas PRE seguidos por (fb) POST para el control de A. rudis GR en soja GFR y sus efectos sobre la densidad y biomasa de $A$. rudis y el rendimiento de la soja. En 2013 y 2014, se realizaron experimentos de campo cerca de Fremont, Nebraska en un campo comercial infestado con $A$. rudis GR. Glufosinate aplicado en POST temprano y tardío brindó $76 \%$ de control de $A$. rudis GR a 14 d después del POST tardío (DALPOST), comparado con $93 \%$ de control con un programa PRE fb POST, cuando se promediaron los tratamientos. Las aplicaciones PRE de chlorimuron más thifensulturon más flumioxazin, $S$-metolachlor más fomesafen o metribuzin, saflufenacil más dimethenamid-P fb glufosinate brindaron $\geq 95 \%$ de control de $A$. rudis a lo largo de la temporada de crecimiento, redujeron la densidad de $A$. rudis a $\leq 2$ plantas $\mathrm{m}^{-2}$, causaron $\geq 94 \%$ de reducción de biomasa, y permitieron
\end{abstract}

\footnotetext{
DOI: $10.1017 /$ wet. 2016.8

* First, second, and third authors: Assistant Professor (ORCID: 0000-0001-8599-4996), Extension Educator, and Postdoctoral Research Associate (ORCID: 0000-0002-1876-8400), Department of Agronomy and Horticulture, University of Nebraska-Lincoln, Lincoln, NE 68583; Fourth author: Associate Professor, West Central Research and Extension Center, University of Nebraska-Lincoln, North Platte, NE 69101; Fifth author: Professor, Northeast Research and Extension Center, Haskell Agricultural Laboratory, University of Nebraska-Lincoln, Concord, NE 68728. Corresponding author's E-mail: Amit.Jhala@unl.edu
} 
un rendimiento de soja de $1,984 \mathrm{a} 2,210 \mathrm{~kg} \mathrm{ha}^{-1}$. Al promediarse los tratamientos, el programa PRE fb POST brindó $82 \%$ de control de $A$. rudis al momento de la cosecha, redujo la densidad a 23 plantas $\mathrm{m}^{-2}$ a 14 DALPOST, causó $86 \%$ de reducción de biomasa, y el rendimiento de la soja fue $1,803 \mathrm{~kg} \mathrm{ha}^{-1}$, comparado con $77 \%$ de control, 99 plantas $\mathrm{m}^{-2}$, $53 \%$ de reducción de biomasa, y un rendimiento de $1,190 \mathrm{~kg} \mathrm{ha}^{-1}$ con el programa de sólo herbicidas POST. Se concluyó que hay programas de herbicidas PRE fb POST disponibles con modos de acción efectivos para el control de $A$. rudis GR en soja GFR.

Common waterhemp, a native to the Great Plains region of the United States, is a problem $\mathrm{C}_{4}$ broadleaf weed species in Nebraska and several other states in the Midwestern United States (Rosenbaum and Bradley 2013; Waselkov and Olsen 2014). Common waterhemp is a prolific seed producer. On average, a single female plant produces 250,000 seeds, though some plants can produce more than 1 million seeds when allowed to grow without competition (Sellers et al. 2003). Common waterhemp is a highly competitive weed that causes significant yield losses in many crops, including corn (Zea mays L.) and soybean (Bensch et al. 2003; Steckel and Sprague 2004). For example, Hager et al. (2002a) reported that when common waterhemp plants were allowed to interfere up to 10 wk after soybean unifoliate expansion, there was a $43 \%$ yield loss in soybean compared with the weedfree control. Steckel and Sprague (2004) reported 74\% corn yield reduction due to season-long common waterhemp interference. Common waterhemp has a prolonged emergence pattern (Refsell and Hartzler 2009), and even late-emerging cohorts have strong seed production potential (Wu and Owen 2014). The species' ability to compete with crops, rapid growth rate, prolific seed production, extended emergence pattern, and ability to thrive under a wide range of stress conditions have established common waterhemp as a successful weed in conventional and no-till crop production systems in the Midwest (Horak and Loughin 2000; Owen 2008; Rosenbaum and Bradley 2013; Sarangi et al. 2016; Steckel et al. 2003; Wu and Owen 2014; 2015).

Since the commercialization of glyphosate-resistant (GR) crops, the continuous use of glyphosate in GR corn and soybean cropping systems and a decline in the use of residual herbicides in the Midwest has resulted in the evolution of GR weeds (Beckie 2006; Culpepper 2006; Young 2006). The first report of a GR weed in the United States was horseweed [Conyza canadensis (L.) Cronq.] in Delaware (VanGessel 2001). As of June 2016, 35 weed species worldwide have been confirmed resistant to glyphosate, including 16 species in the United States (Heap 2016a) and six in Nebraska
(Jhala 2016). The first report of GR common waterhemp was in Missouri in 2008 (Legleiter and Bradley 2008), and as of 2016, it has been confirmed in 17 states in the United States (Heap 2016b) and in Ontario, Canada (P. Sikkema, personal communication). Common waterhemp biotypes resistant to herbicides belonging to other mode of action groups have also been confirmed. For example, common waterhemp populations resistant to acetolactate synthase (ALS)-inhibitors (Horak and Peterson 1995), photosystem II-inhibitors (Anderson et al. 1996), protoporphyrinogen oxidase (PPO)-inhibitors (Shoup et al. 2003), 4-hydroxyphenylpyruvate dioxygenase (HPPD)-inhibitors (Hausman et al. 2011), and synthetic auxins (Bernards et al. 2012) have been reported. Common waterhemp resistant to multiple herbicides has also been reported (Bell et al. 2013; Legleiter and Bradley 2008). Glyphosate-resistant common waterhemp has recently been confirmed in several eastern Nebraska counties (Sarangi et al. 2015), and management of GR common waterhemp has become a challenge for Nebraska corn and soybean growers. Additionally, the majority of GR common waterhemp biotypes in eastern Nebraska have decreased sensitivity to ALS-inhibiting herbicides, further lowering the number of effective POST herbicide options for management in GR soybean (Sarangi et al. 2015).

Glufosinate is a contact, POST herbicide for control of a broad spectrum of emerged broadleaf and grassy weeds. It is a non-selective herbicide historically used for weed control in fruit and nut orchards and non-crop areas; however, after the commercialization of glufosinate-resistant (GFR) crops in 1999, glufosinate has been used POST in crops resistant to glufosinate, including soybean (Wiesbrook et al. 2001). Glufosinate inhibits glutamine synthetase, an enzyme that is essential for nitrogen metabolism in plants (Logusch et al. 1991). Glutamine synthetase is involved in the assimilation of ammonium, and inhibition of this enzyme results in the buildup of ammonium in plant tissue, indirectly inhibiting photorespiration and photosynthesis in the plant and thus causing plant death (Wild and Manderscheid 1984). Though the 
adoption of GFR crops has been slow, the evolution of GR weeds is causing growers to search for alternative herbicide-resistant cropping technologies (Aulakh and Jhala 2015). For example, growers began to adopt GFR soybean in the Mid-South as an option for controlling GR Palmer amaranth (Amaranthus palmeri S. Wats) (Riar et al. 2013). It is possible that GFR soybean will be adopted on a relatively large scale in the Midwest in the near future for the control of GR weeds, including common waterhemp. Research conducted in Nebraska reported excellent control of GR giant ragweed (Ambrosia trifida L.) and GR volunteer corn in GFR soybean (Chahal and Jhala 2015; Kaur et al. 2014). More information is needed to develop recommendations for herbicide programs that can provide effective control of GR common waterhemp and other difficultto-control weeds in GFR soybean.

Glufosinate can be applied in a single application or sequentially, though its maximum cumulative total may not exceed $1,329 \mathrm{~g}$ ai ha ${ }^{-1}$ per growing season in GFR soybean (Anonymous 2016). If applied in a burndown (before planting) program, the application rate can be 593 to $736 \mathrm{~g}_{\text {ai ha }}{ }^{-1}$, with an additional in-season application of $593 \mathrm{~g}$ ai ha ${ }^{-1}$ before but not during the bloom growth stage of GFR soybean (Anonymous 2016). Sequential applications of glufosinate should be made at least five days apart. Aulakh and Jhala (2015) reported $<82 \%$ control of common waterhemp, common lambsquarters (Chenopodium album L.), and eastern black nightshade (Solanum ptychanthum Dunal) with glufosinate applied early and late POST, compared with $\geq 95 \%$ control with sulfentrazone plus metribuzin applied PRE followed by $(\mathrm{fb})$ glufosinate plus pyroxasulfone (3-[[5-(difluoromethoxy)-1-methyl-3(trifluoromethyl)pyrazol-4-yl] methylsulfonyl]-5,5dimethyl-4H-1,2-oxazole) or acetochlor applied POST. Similarly, Bell et al. (2016) reported 98\% control of GR Palmer amaranth in GFR soybean with flumioxazin plus pyroxasulfone applied PRE $\mathrm{fb}$ glufosinate, but $<70 \%$ control with glufosinate applied sequentially. Therefore, it is important to incorporate residual herbicides with different modes of action in glufosinate-based herbicide programs to achieve season-long control of GR weeds such as common waterhemp.

Scientific literature comparing one- and two-pass POST herbicide programs to PRE fb POST programs for controlling GR common waterhemp in GFR soybean is limited. The objective of this study was to compare glufosinate-based one- or two-pass POST herbicide (POST-only) programs to PRE $\mathrm{fb}$ POST programs for the management of GR common waterhemp. We evaluated the effect of each treatment on common waterhemp density and biomass and GFR soybean injury and yield. We hypothesized that residual PRE herbicides applied at planting fb glufosinate would provide better control of GR common waterhemp and higher soybean yield than POST-only programs.

\section{Materials and Methods}

Field Experiments. Field experiments were conducted during the summers of 2013 and 2014 in a grower's field near Fremont, NE $\left(41.47^{\circ} \mathrm{N}, 96.46^{\circ} \mathrm{W}\right)$ that was infested with GR common waterhemp. The level of glyphosate resistance in the common waterhemp biotype from this site was 16- to 24-fold that of known susceptible biotypes, and it also had a reduced susceptibility to ALS-inhibiting herbicides (Sarangi et al. 2015). Common waterhemp was the dominant weed at the research site, with an average density of 250 to 300 plants $\mathrm{m}^{-2}$. The field had been under GR corn or soybean production systems with a reliance on glyphosate for weed control for at least $8 \mathrm{yr}$. The soil at the experimental site was clay (Luton series) with a $\mathrm{pH}$ of 6.7 , and comprised $29 \%$ sand, $30 \%$ silt, $41 \%$ clay, and $4 \%$ organic matter. A soybean cultivar resistant to glufosinate was planted in a conventionally-tilled seedbed at 345,000 seeds ha ${ }^{-1}$ in rows spaced $76.2 \mathrm{~cm}$ apart. Soybean was planted on June 11 in 2013, due to adverse weather conditions early in the season, and on May 20 in 2014. Individual plots measured $3 \mathrm{~m}$ wide by $9 \mathrm{~m}$ long. The experimental site was located in a rainfed, dryland environment with no supplemental irrigation; however, precipitation was adequate to activate the residual herbicides (Table 1).

Field experiments were arranged in a randomized complete block design with four replications for each treatment. The herbicide programs evaluated to control GR common waterhemp consisted of one-pass POST, two-pass POST, and PRE $\mathrm{fb}$ POST programs (Table 2). A non-treated control was included for comparison. Herbicides were applied with a handheld, $\mathrm{CO}_{2}$-pressurized backpack sprayer equipped with AIXR 110015 flat fan nozzles (TeeJet ${ }^{\circledR}$ Technologies, Spraying Systems Co., P.O. Box 7900, Wheaton, IL 60187 ) calibrated to deliver $140 \mathrm{~L} \mathrm{ha}^{-1}$ at $276 \mathrm{kPa}$ at a constant speed of $4.8 \mathrm{~km} \mathrm{~h}^{-1}$. To improve efficacy, 
Table 1. Monthly mean air temperature and total precipitation during the 2013 and 2014 growing seasons, along with the 30-yr average, at Fremont, Nebraska. ${ }^{\mathrm{a}}$

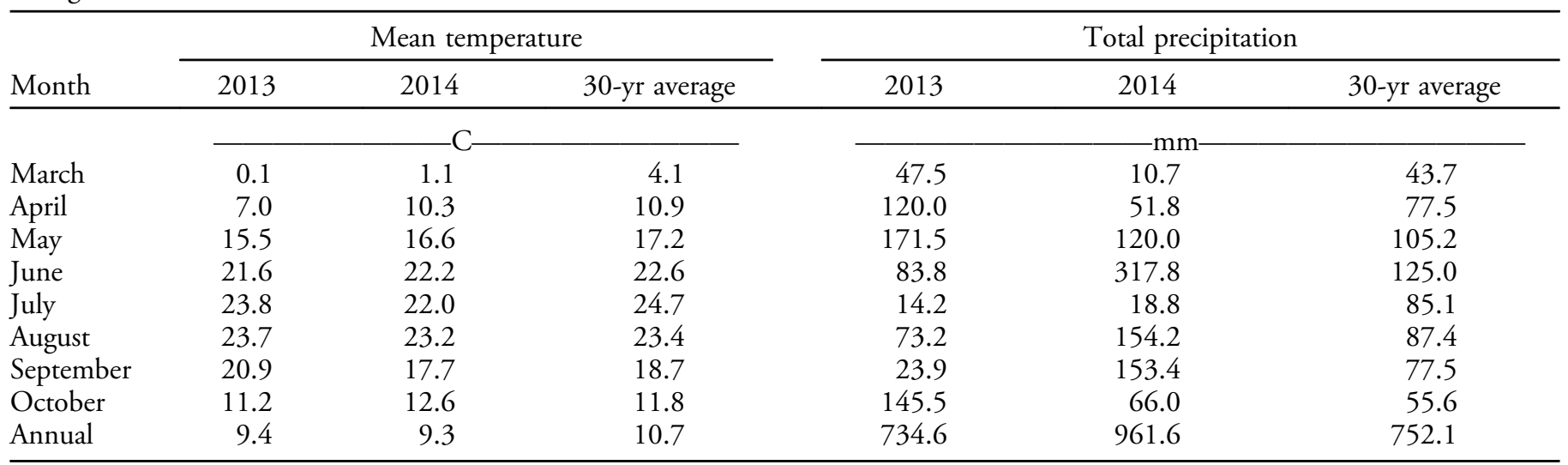

${ }^{\mathrm{a}}$ Data were obtained from National Oceanic and Atmospheric Administration (NOAA 2015).

each treatment with glufosinate was mixed with ammonium sulfate at $3.4 \mathrm{~kg} \mathrm{ha}^{-1}$, as recommended on the label (Anonymous 2016). PRE herbicides were applied on the day of soybean planting, whereas earlyPOST herbicides were applied $21 \mathrm{~d}$ after PRE (DAPRE), at which time the common waterhemp was 8 to $18 \mathrm{~cm}$ tall (depending on treatment), and soybean was at the first to second trifoliate stage. LatePOST herbicide applications were made $14 \mathrm{~d}$ after the early-POST herbicide applications (DAEPOST), when common waterhemp plants were 5 to $20 \mathrm{~cm}$ tall. Common waterhemp plant height at the time of latePOST herbicide application was variable because some new plants had emerged and some plants had been partially controlled by the early-POST herbicide applications.

Data Collection. Common waterhemp control was assessed visually at 14 DAPRE, 14 DAEPOST, $14 \mathrm{~d}$ after late-POST (DALPOST) herbicide applications, and at soybean harvest, on a scale of $0 \%$ to $100 \%$, with $0 \%$ meaning no control or injury symptoms on common waterhemp plants, and $100 \%$ meaning complete control. Common waterhemp densities were recorded at 14 DAPRE and 14 DALPOST by counting the number of common waterhemp plants in two $0.25 \mathrm{~m}^{2}$ quadrats placed randomly between the center two soybean rows in each plot and were reported as the number of plants per square meter. At 28 DALPOST, common waterhemp plants that survived the herbicide treatments were cut at the soil surface from two randomly selected $0.25 \mathrm{~m}^{2}$ quadrats per plot and oven-dried at $65 \mathrm{C}$ until they reached a constant weight. Aboveground biomass was converted into percent biomass reduction compared with the non-treated control using the following equation (Wortman 2014):

$$
\% \text { biomass reduction }=[(\bar{C}-B) / \bar{C}] \times 100
$$

where $\bar{C}$ is the biomass of the non-treated control and $B$ is the biomass of an individual treated plot. Soybean injury data were recorded at 14 DAPRE, 7 DAEPOST, 7 DALPOST, and 28 DALPOST, on a scale of $0 \%$ to $100 \%$, with $0 \%$ indicating no soybean injury and $100 \%$ indicating death of soybean plants. Soybean was harvested from the center two rows in each plot using a plot combine (Gleaner K2; AGCO, 4205 River Green Parkway, Duluth, GA). The combine had a row-crop header that can harvest two rows that are $76 \mathrm{~cm}$ apart, and included the HarvestMaster System equipped with Mirus Data collection software (Juniper Systems \& HarvestMaster, Logan, UT) for determining seed weight. Grain yield was adjusted to $13 \%$ moisture content.

Statistical Analysis. Data were subjected to ANOVA using the PROC GLIMMIX procedure in $\mathrm{SAS}^{\circledR}$ version 9.3 (SAS Institute Inc., Cary, NC). In the model, years and treatments were considered fixed effects, whereas blocks, which were nested within years, were considered random effects. Data were tested for normality using PROC UNIVARIATE. Common waterhemp visual control estimates and percent biomass reduction data were arcsine square root transformed before analysis; however, back-transformed data are presented with mean separation based on transformed data. Individual treatment means were separated at the $5 \%$ level of significance using Fisher's protected 
Table 2. Details of herbicide treatments, application timings, and rates used for control of glyphosate-resistant common waterhemp in glufosinate-resistant soybean, in field experiments conducted in Nebraska in 2013 and 2014.

\begin{tabular}{|c|c|c|c|c|}
\hline Herbicide program $^{\mathrm{a}}$ & Trade name & Application timing & Rate & Manufacturer \\
\hline $\begin{array}{l}\text { Glufosinate }+ \text { fomesafen }+ \\
\text { imazethapyr }+ \text { acetochlor }\end{array}$ & $\begin{array}{l}\text { Liberty + Flexstar + } \\
\text { Pursuit + Warrant }\end{array}$ & Early POST & $\begin{array}{c}\text { g ae or ai ha-1 } \\
594+263+70+1,680\end{array}$ & $\begin{array}{l}\text { Bayer CropScience, Research Triangle Park, } \\
\text { NC + Syngenta Crop Protection, } \\
\text { Greensboro, NC + BASF Corporation, } \\
\text { Research Triangle Park, NC + Monsanto } \\
\text { Company, St. Louis, MO }\end{array}$ \\
\hline Glufosinate $\mathrm{fb}$ & Liberty $\mathrm{fb}$ & Early POST fb & 594 & Bayer CropScience \\
\hline glufosinate & Liberty & late POST & 594 & Bayer CropScience \\
\hline $\begin{array}{l}\text { Glufosinate }+ \text { acetochlor } \mathrm{fb} \\
\text { glufosinate }\end{array}$ & $\begin{array}{l}\text { Liberty + Warrant fb } \\
\text { Liberty }\end{array}$ & $\begin{array}{l}\text { Early POST fb } \\
\text { late POST }\end{array}$ & $\begin{array}{l}594+1,680 \\
594\end{array}$ & $\begin{array}{l}\text { Bayer CropScience + Monsanto Company, } \\
\text { Bayer CropScience }\end{array}$ \\
\hline $\begin{array}{l}\text { Glufosinate + acetochlor + imazethapyr fb } \\
\text { glufosinate }\end{array}$ & $\begin{array}{l}\text { Liberty + Warrant }+ \text { Pursuit } \mathrm{fb} \\
\text { Liberty }\end{array}$ & $\begin{array}{l}\text { Early POST fb } \\
\text { late POST }\end{array}$ & $\begin{array}{l}594+1,680+70 \\
594\end{array}$ & $\begin{array}{l}\text { Bayer CropScience }+ \text { Monsanto Company }+ \\
\text { BASF Corporation fb Bayer CropScience }\end{array}$ \\
\hline $\begin{array}{l}\text { Glufosinate }+ \text { fomesafen } \mathrm{fb} \\
\text { glufosinate }\end{array}$ & $\begin{array}{l}\text { Liberty }+ \text { Flexstar fb } \\
\text { Liberty }\end{array}$ & $\begin{array}{l}\text { Early POST fb } \\
\text { late POST }\end{array}$ & $\begin{array}{l}594+263 \\
594\end{array}$ & $\begin{array}{l}\text { Bayer CropScience }+ \text { Syngenta Crop } \\
\text { Protection, Bayer CropScience }\end{array}$ \\
\hline $\begin{array}{l}\text { Glufosinate }+ \text { fomesafen }+ \text { acetochlor } \mathrm{fb} \\
\text { glufosinate }\end{array}$ & $\begin{array}{l}\text { Liberty }+ \text { Flexstar }+ \text { Warrant fb } \\
\text { Liberty }\end{array}$ & $\begin{array}{l}\text { Early POST fb } \\
\text { late POST }\end{array}$ & $\begin{array}{l}594+263+1,680 \\
594\end{array}$ & $\begin{array}{l}\text { Bayer CropScience + Syngenta Crop } \\
\text { Protection + Monsanto Company fb Bayer } \\
\text { CropScience LP }\end{array}$ \\
\hline $\begin{array}{l}\text { Glufosinate }+ \text { fomesafen }+ \text { imazethapyr }+ \\
\text { acetochlor } \mathrm{fb} \\
\text { glufosinate }\end{array}$ & $\begin{array}{l}\text { Liberty }+ \text { Flexstar }+ \text { Pursuit }+ \\
\text { Warrant } \mathrm{fb} \\
\text { Liberty }\end{array}$ & $\begin{array}{c}\text { Early POST fb } \\
\text { late POST }\end{array}$ & $594+263+70+1,680$ & $\begin{array}{l}\text { Bayer CropScience + Syngenta Crop } \\
\text { Protection + BASF Corporation }+ \\
\text { Monsanto Company fb Bayer CropScience }\end{array}$ \\
\hline $\begin{array}{l}\text { Flumioxazin } \mathrm{fb} \\
\text { glufosinate }\end{array}$ & $\begin{array}{l}\text { Valor } \mathrm{fb} \\
\text { Liberty }\end{array}$ & $\begin{array}{l}\text { PRE fb } \\
\text { late POST }\end{array}$ & $\begin{array}{c}107 \\
594\end{array}$ & $\begin{array}{l}\text { Valent USA Corporation, Walnut Creek, CA } \\
\text { Bayer CropScience }\end{array}$ \\
\hline $\begin{array}{l}\text { Flumioxazin + cloransulam-methyl fb } \\
\text { glufosinate }\end{array}$ & $\begin{array}{l}\text { Valor + FirstRate } \mathrm{fb} \\
\text { Liberty }\end{array}$ & $\begin{array}{l}\text { PRE fb } \\
\text { late POST }\end{array}$ & $\begin{array}{l}107+35.3 \\
594\end{array}$ & $\begin{array}{l}\text { Valent + Dow AgroSciences, Indianapolis, IN } \\
\text { Bayer CropScience }\end{array}$ \\
\hline $\begin{array}{l}\text { Chlorimuron-ethyl + thifensulfuron- } \\
\text { methyl + flumioxazin } \mathrm{fb}\end{array}$ & Envive $\mathrm{fb}$ & PRE fb & 153 & $\begin{array}{l}\text { E. I. du Pont de Nemours and Company, } \\
\text { Wilmington, DE }\end{array}$ \\
\hline glufosinate & Liberty & late POST & 594 & Bayer CropScience \\
\hline $\begin{array}{l}\text { Alachlor fb } \\
\text { glufosinate }\end{array}$ & $\begin{array}{l}\text { Intrro fb } \\
\text { Liberty }\end{array}$ & $\begin{array}{l}\text { PRE fb } \\
\text { late POST }\end{array}$ & $\begin{array}{l}3,360 \\
594\end{array}$ & $\begin{array}{l}\text { Monsanto Company fb } \\
\text { Bayer CropScience }\end{array}$ \\
\hline $\begin{array}{l}S \text {-metolachlor } \mathrm{fb} \\
\text { glufosinate }\end{array}$ & $\begin{array}{l}\text { Dual II Magnum fb } \\
\text { Liberty }\end{array}$ & $\begin{array}{l}\text { PRE fb } \\
\text { late POST }\end{array}$ & $\begin{array}{l}1420 \\
594\end{array}$ & $\begin{array}{l}\text { Syngenta Crop Protection } \\
\text { Bayer CropScience }\end{array}$ \\
\hline $\begin{array}{l}S \text {-metolachlor + imazethapyr fb } \\
\text { glufosinate }\end{array}$ & $\begin{array}{l}\text { Dual II Magnum }+ \text { Pursuit } \mathrm{fb} \\
\text { Liberty }\end{array}$ & $\begin{array}{l}\text { PRE fb } \\
\text { late POST }\end{array}$ & $\begin{array}{l}1,420+70 \\
594\end{array}$ & $\begin{array}{l}\text { Syngenta Crop Protection + BASF Corporation } \\
\text { Bayer CropScience }\end{array}$ \\
\hline $\begin{array}{l}S \text {-metolachlor }+ \text { fomesafen } \mathrm{fb} \\
\text { glufosinate }\end{array}$ & $\begin{array}{l}\text { Prefix fb } \\
\text { Liberty }\end{array}$ & $\begin{array}{l}\text { PRE fb } \\
\text { late POST }\end{array}$ & $\begin{array}{l}1,480 \\
594\end{array}$ & $\begin{array}{l}\text { Syngenta Crop Protection } \\
\text { Bayer CropScience }\end{array}$ \\
\hline $\begin{array}{l}\text { S-metolachlor + metribuzin } \mathrm{fb} \\
\text { glufosinate }\end{array}$ & $\begin{array}{l}\text { Boundary } \mathrm{fb} \\
\text { Liberty }\end{array}$ & $\begin{array}{l}\text { PRE fb } \\
\text { late POST }\end{array}$ & $\begin{array}{l}2,050 \\
594\end{array}$ & $\begin{array}{l}\text { Syngenta Crop Protection } \\
\text { Bayer CropScience }\end{array}$ \\
\hline
\end{tabular}




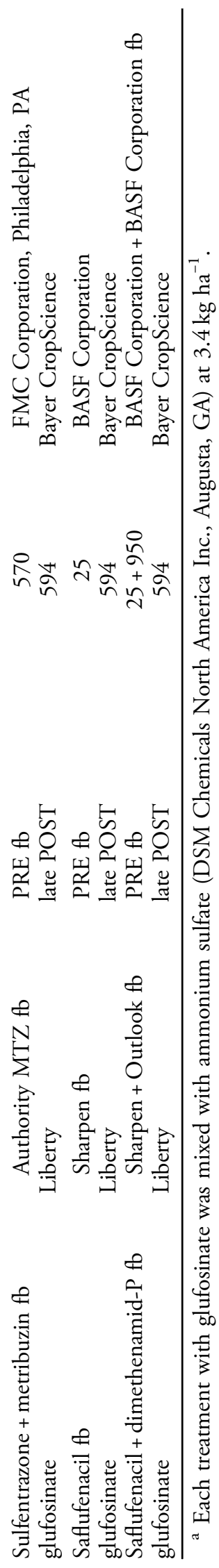

LSD test. To determine relative treatment efficacy for common waterhemp control, density, biomass reduction, and soybean yield, a priori orthogonal contrasts (single degree of freedom) were performed. Preplanned contrasts were conducted to compare one-pass POST to two-pass POST, and to compare POST-only to the PRE fb POST programs.

\section{Results and Discussion}

Year-by-treatment interactions for GR common waterhemp control estimates, density, and biomass, and soybean yield were not significant; therefore, data from both years were combined.

Common Waterhemp Control. Two-pass herbicide programs provided $78 \%$ control of common waterhemp, compared with $93 \%$ control with PRE fb POST programs, when averaged across treatments at 14 DALPOST (Table 3), indicating the importance of residual herbicides $\mathrm{fb}$ a late-POST glufosinate application for common waterhemp control. Two-pass POST herbicide programs provided $77 \%$ control, while the PRE $\mathrm{fb}$ POST program provided 90\% control, when averaged across treatments at 14 DAEPOST. This is due to the excellent control of common waterhemp that can be achieved with residual herbicides applied PRE with a follow-up application of glufosinate when plants are less than $12 \mathrm{~cm}$ tall. Krausz and Young (2003) also reported 89\% to 99\% control of common waterhemp with sulfentrazone-based tankmixtures applied PRE fb glyphosate in GR soybean.

Herbicides applied PRE resulted in $76 \%$ to $99 \%$ control of GR common waterhemp at 14 DAPRE (Table 4). Similarly, Aulakh and Jhala (2015) reported $\geq 92 \%$ control of common waterhemp and common lambsquarters at 15 DAPRE, but no control using a POST-only herbicide program. Among PRE herbicides, flumioxazin plus cloransulam, chlorimuron plus thifensulfuron plus flumioxazin, $S$-metolachlor plus fomesafen, $S$-metolachlor or sulfentrazone plus metribuzin, and saflufenacil plus dimethenamid provided $\geq 98 \%$ control. Similar to the results of this study, Bell et al. (2015) reported $>99 \%$ control of Palmer amaranth $21 \mathrm{~d}$ after soybean planting when $S$-metolachlor plus metribuzin was applied PRE at the time of planting.

At 14 DAEPOST, the POST-only programs that we tested provided $71 \%$ to $82 \%$ control of common waterhemp (Table 5), while the PRE fb POST herbicide programs provided $71 \%$ to $99 \%$ control. 
Table 3. Orthogonal contrasts ${ }^{a}$ for comparison of herbicide programs for glyphosate-resistant common waterhemp control, density, and biomass reduction and soybean yield in field experiments conducted near Fremont, Nebraska in 2013 and 2014 . $^{\mathrm{b}}$

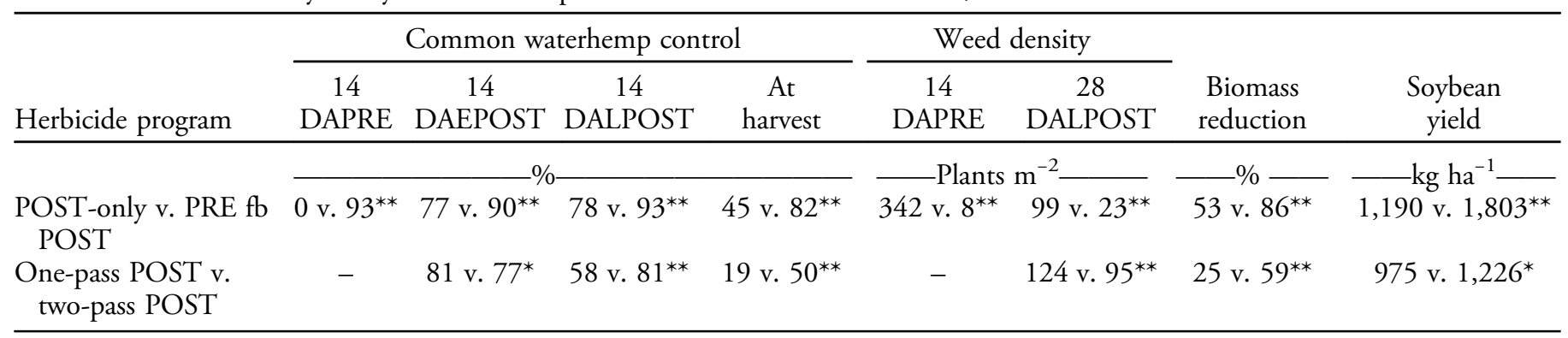

${ }^{\text {a }}$ a priori orthogonal contrasts.

b Abbreviations: DAEPOST, days after early-post-emergence herbicide treatment; DAPRE, days after pre-emergence herbicide treatment; DALPOST, days after late-post-emergence herbicide treatment; v., versus.

${ }^{*}$ Significant at $P<0.05$; ${ }^{* *}$ significant at $P<0.01$.

This is likely due to the fact that when early-POST herbicides were applied, common waterhemp plants were 8 to $18 \mathrm{~cm}$ tall with a density of approximately 300 plants $\mathrm{m}^{-2}$, and therefore were less likely to be effectively controlled with a glufosinate-based herbicide program because the efficacy of glufosinate can be affected by weed height and density. For instance, Barnett et al. (2013) reported $>90 \%$ control of Palmer amaranth when glufosinate was applied to $13-\mathrm{cm}$-tall plants, but $<60 \%$ control when glufosinate was applied to 26-cm-tall plants. In a bare-ground study in Illinois, Steckel et al. (1997) reported $>80 \%$ control of giant foxtail (Setaria faberi Herrm.), common lambsquarters, common cocklebur (Xanthium strumarium L.), and Pennsylvania smartweed (Polygonum pensylvanicum L.) when glufosinate was applied at the $10-\mathrm{cm}$ weed height, but $<70 \%$ control at the $15-\mathrm{cm}$ weed height. In contrast, Coetzer et al. (2002) reported $82 \%$ to $87 \%$ control at $4 \mathrm{wk}$ after treatment with glufosinate applied alone at $410 \mathrm{~g}$ ai ha ${ }^{-1}$ when common waterhemp plants ranged from 2 to $18 \mathrm{~cm}$ tall.

Glufosinate applied alone resulted in $71 \%$ control, while glufosinate tank-mixed with acetochlor, imazethapyr, and/or fomesafen resulted in $75 \%$ to $82 \%$ control (Table 5). Similarly, Chahal and Johnson (2012) reported $78 \%$ to $84 \%$ control of GR common lambsquarters with glufosinate tank-mixed with 2,4-D or dicamba, but only $44 \%$ control with glufosinate applied alone. Aulakh and Jhala (2015) reported $<73 \%$ control of common waterhemp with glufosinate applied alone in GFR soybean. Among POST-only herbicide programs, a one-pass POST application of glufosinate plus fomesafen plus acetochlor plus imazethapyr, a program with four distinct modes of action, provided $58 \%$ control at 14 DALPOST, while two-pass POST herbicide programs provided $76 \%$ to $83 \%$ control (Table 5). These data suggest that including herbicides with multiple modes of action is not sufficient to achieve a high level of common waterhemp control, and that the application timing is critical. For example, at least five PRE fb POST herbicide programs with multiple modes of action provided $97 \%$ to $99 \%$ control at 14 DALPOST in this study (Table 4). Similarly, Bell et al. (2016) reported $\geq 95 \%$ control of Palmer amaranth with flumioxazin plus pyroxasulfone applied PRE in GR and GFR soybean. Flumioxazin applied alone and $S$-metolachlor plus imazethapyr provided $<62 \%$ control at harvest.

Averaged across treatments, a two-pass POST program provided $50 \%$ control of GR common waterhemp at harvest, while a one-pass POST program provided $19 \%$ control, indicating the failure of one- or two-pass POST herbicide programs to effectively control GR common waterhemp (Table 3). Aulakh and Jhala (2015) also reported $65 \%$ to $81 \%$ control of common waterhemp with glufosinate-based one- or two-pass POST programs in GFR soybean. Contrast analysis of common waterhemp control estimates at soybean harvest suggest $82 \%$ control with PRE $\mathrm{fb}$ POST herbicide programs compared with $45 \%$ control with POST-only programs. In a study conducted in Nebraska, Sarangi (2016) reported 84\% control of GR common waterhemp at soybean harvest in a PRE $\mathrm{fb}$ POST program, but only $42 \%$ control with a POST-only program. We found that PRE fb POST programs using chlorimuron plus thifensulfuron plus flumioxazin, $S$-metolachlor plus fomesafen or 
Table 4. Effect of PRE followed by POST herbicide programs on glyphosate-resistant common waterhemp control in glufosinateresistant soybean at $14 \mathrm{~d}$ after PRE herbicide application, $14 \mathrm{~d}$ after early-POST herbicide application, $14 \mathrm{~d}$ after late-POST herbicide application, and at soybean harvest, in field experiments conducted near Fremont, Nebraska in 2013 and 2014. a,b

\begin{tabular}{|c|c|c|c|c|c|c|}
\hline \multirow[b]{2}{*}{ Herbicide program } & \multirow[b]{2}{*}{ Application timing } & \multirow[b]{2}{*}{ Rate } & \multicolumn{4}{|c|}{ Common waterhemp control ${ }^{c, d}$} \\
\hline & & & 14 DAPRE & 14 DAEPOST & 14 DALPOST & At harvest \\
\hline & & $\mathrm{g}$ ae or ai ha ${ }^{-1}$ & & & & \\
\hline $\begin{array}{l}\text { Flumioxazin } \mathrm{fb} \\
\text { glufosinate }\end{array}$ & $\begin{array}{l}\text { PRE fb } \\
\text { late POST }\end{array}$ & $\begin{array}{l}107 \\
594\end{array}$ & $88 \mathrm{c}$ & $83 \mathrm{~d}$ & $88 \mathrm{c}$ & 59 e \\
\hline $\begin{array}{l}\text { Flumioxazin + cloransulam fb } \\
\text { glufosinate }\end{array}$ & $\begin{array}{l}\text { PRE fb } \\
\text { late POST }\end{array}$ & $\begin{array}{c}107+35.3 \\
594\end{array}$ & $99 \mathrm{a}$ & $96 \mathrm{bc}$ & $97 \mathrm{ab}$ & $87 \mathrm{~cd}$ \\
\hline $\begin{array}{l}\text { Chlorimuron + thifensulfuron + } \\
\text { flumioxazin } \mathrm{fb}\end{array}$ & PRE fb & 153 & $99 \mathrm{a}$ & $98 \mathrm{ab}$ & $99 \mathrm{a}$ & $96 \mathrm{ab}$ \\
\hline glufosinate & $\begin{array}{l}\text { late POST } \\
\text { PRF fh }\end{array}$ & 594 & & & & \\
\hline $\begin{array}{l}\text { Alachlor fb } \\
\text { glufosinate }\end{array}$ & $\begin{array}{l}\text { PRE fb } \\
\text { late POST }\end{array}$ & $\begin{array}{c}3,360 \\
594\end{array}$ & $96 \mathrm{~b}$ & $93 \mathrm{c}$ & $96 \mathrm{~b}$ & $86 \mathrm{~cd}$ \\
\hline $\begin{array}{l}S \text {-metolachlor fb } \\
\text { glufosinate }\end{array}$ & $\begin{array}{l}\text { PRE fb } \\
\text { late POST }\end{array}$ & $\begin{array}{l}1,420 \\
594\end{array}$ & $76 \mathrm{~d}$ & $71 \mathrm{e}$ & $80 \mathrm{~d}$ & $54 \mathrm{e}$ \\
\hline $\begin{array}{l}S \text {-metolachlor + imazethapyr fb } \\
\text { glufosinate }\end{array}$ & $\begin{array}{l}\text { PRE fb } \\
\text { late POST }\end{array}$ & $\begin{array}{l}1,420+70 \\
594\end{array}$ & $78 \mathrm{~d}$ & $74 \mathrm{e}$ & $81 \mathrm{~d}$ & $61 \mathrm{e}$ \\
\hline $\begin{array}{l}\text { S-metolachlor }+ \text { fomesafen } \mathrm{fb} \\
\text { glufosinate }\end{array}$ & $\begin{array}{l}\text { PRE fb } \\
\text { late POST }\end{array}$ & $\begin{array}{l}1,480 \\
594\end{array}$ & $99 \mathrm{a}$ & $99 \mathrm{a}$ & $99 \mathrm{a}$ & $98 \mathrm{a}$ \\
\hline $\begin{array}{l}\text { S-metolachlor + metribuzin fb } \\
\text { glufosinate }\end{array}$ & $\begin{array}{l}\text { PRE fb } \\
\text { late POST }\end{array}$ & $\begin{array}{c}2,050 \\
594\end{array}$ & $99 \mathrm{a}$ & $98 \mathrm{ab}$ & $99 \mathrm{a}$ & $95 \mathrm{ab}$ \\
\hline $\begin{array}{l}\text { Sulfentrazone + metribuzin } \mathrm{fb} \\
\text { glufosinate }\end{array}$ & $\begin{array}{l}\text { PRE fb } \\
\text { late POST }\end{array}$ & $\begin{array}{l}570 \\
594\end{array}$ & $98 \mathrm{ab}$ & $96 \mathrm{bc}$ & $96 \mathrm{~b}$ & $91 \mathrm{bc}$ \\
\hline $\begin{array}{l}\text { Saflufenacil fb } \\
\text { glufosinate }\end{array}$ & $\begin{array}{l}\text { PRE fb } \\
\text { late POST }\end{array}$ & $\begin{array}{c}25 \\
594\end{array}$ & $90 \mathrm{c}$ & $87 \mathrm{~d}$ & $92 \mathrm{c}$ & $83 \mathrm{~d}$ \\
\hline $\begin{array}{l}\text { Saflufenacil + dimethenamid fb } \\
\text { glufosinate }\end{array}$ & $\begin{array}{l}\text { PRE fb } \\
\text { late POST }\end{array}$ & $\begin{array}{l}25+950 \\
594\end{array}$ & $99 \mathrm{a}$ & $99 \mathrm{a}$ & $99 \mathrm{a}$ & $97 \mathrm{a}$ \\
\hline$P$-value & & & $<0.0001$ & $<0.0001$ & $<0.0001$ & $<0.0001$ \\
\hline
\end{tabular}

${ }^{a}$ Year-by-treatment interaction for glyphosate-resistant common waterhemp control was not significant; therefore, data were combined across the two years.

b Abbreviations: DAEPOST, $\mathrm{d}$ after early-POST herbicide application; DALPOST, $\mathrm{d}$ after late-POST herbicide application; DAPRE, $\mathrm{d}$ after PRE herbicide application; fb, followed by.

${ }^{\mathrm{c}}$ Data were arcsine square root transformed before analysis; however, back-transformed original mean values are presented with the interpretation from the transformed data.

${ }^{\mathrm{d}}$ Means presented within each column with no common letter(s) are significantly different according to Fisher's protected LSD test at $P \leq 0.05$.

metribuzin, and saflufenacil plus dimethenamid $\mathrm{fb}$ glufosinate applied POST provided $\geq 95 \%$ control at soybean harvest. In a 2-yr study in Arkansas, Bell et al. (2015) observed $86 \%$ to $95 \%$ Palmer amaranth control at harvest in GFR soybean with $S$-metolachlor plus metribuzin applied PRE fb glufosinate applied POST, but only $50 \%$ to $85 \%$ control with a POST-only program. The results of this study are consistent with several previous studies in suggesting that PRE fb POST programs are better for control of Amaranthus than POST-only programs (Aulakh and Jhala 2015; Bell et al. 2015; 2016; Butts et al. 2016; Hager et al. 2002b; Jhala et al. 2015; Johnson et al. 2012; Meyer et al. 2015; Norsworthy et al. 2012; Sarangi 2016).
Common Waterhemp Density and Biomass.

Common waterhemp density and biomass were both affected by the herbicide programs evaluated (Tables 6 and 7). At 14 DAPRE, plots that received PRE herbicides had common waterhemp densities as low as 0 to 34 plants $\mathrm{m}^{-2}$. Similarly, Sarangi (2016) reported common waterhemp density of $<35$ plants $\mathrm{m}^{-2}$ at $21 \mathrm{~d}$ after PRE herbicide application compared with 323 to 391 plants $\mathrm{m}^{-2}$ with a POSTonly program. Aulakh and Jhala (2015) also reported 0 to 6 common waterhemp plants $\mathrm{m}^{-2}$ with several PRE programs, compared with 11 to 12 plants $\mathrm{m}^{-2}$ in a POST-only program $15 \mathrm{~d}$ after POST herbicides were applied. 
Table 5. Effect of POST-only herbicide programs on glyphosate-resistant common waterhemp control in glufosinate-resistant soybean at $14 \mathrm{~d}$ after early-POST herbicide application, $14 \mathrm{~d}$ after late-POST herbicide application, and at soybean harvest, in field experiments conducted near Fremont, Nebraska in 2013 and 2014.,

\begin{tabular}{|c|c|c|c|c|c|}
\hline \multirow[b]{2}{*}{ Herbicide program } & \multirow[b]{2}{*}{ Application timing } & \multirow[b]{2}{*}{ Rate } & \multicolumn{3}{|c|}{ Common waterhemp control ${ }^{c, d}$} \\
\hline & & & 14 DAEPOST & 14 DALPOST & At harvest ${ }^{c, d}$ \\
\hline & & $\mathrm{g}$ ae or ai $\mathrm{ha}^{-1}$ & & - $\%$ & \\
\hline $\begin{array}{l}\text { Glufosinate + fomesafen }+ \\
\text { imazethapyr + acetochlor }\end{array}$ & Early POST & $594+263+70+1,680$ & $81 \mathrm{a}$ & $58 \mathrm{c}$ & $19 \mathrm{c}$ \\
\hline $\begin{array}{l}\text { Glufosinate } \mathrm{fb} \\
\text { glufosinate }\end{array}$ & $\begin{array}{l}\text { Early POST fb } \\
\text { late POST }\end{array}$ & $\begin{array}{l}594 \\
594\end{array}$ & $71 \mathrm{c}$ & $76 \mathrm{~b}$ & $41 \mathrm{~b}$ \\
\hline $\begin{array}{l}\text { Glufosinate }+ \text { acetochlor } \mathrm{fb} \\
\text { glufosinate }\end{array}$ & $\begin{array}{l}\text { Early POST fb } \\
\text { late POST }\end{array}$ & $\begin{array}{l}594+1,680 \\
594\end{array}$ & $77 \mathrm{ab}$ & $81 \mathrm{ab}$ & $53 \mathrm{a}$ \\
\hline $\begin{array}{l}\text { Glufosinate + acetochlor + imazethapyr fb } \\
\text { glufosinate }\end{array}$ & $\begin{array}{l}\text { Early POST fb } \\
\text { late POST }\end{array}$ & $\begin{array}{l}594+1,680+70 \\
594\end{array}$ & $77 \mathrm{ab}$ & $81 \mathrm{ab}$ & $54 \mathrm{a}$ \\
\hline $\begin{array}{l}\text { Glufosinate + fomesafen } \mathrm{fb} \\
\text { glufosinate }\end{array}$ & $\begin{array}{l}\text { Early POST fb } \\
\text { late POST }\end{array}$ & $\begin{array}{l}594+263 \\
594\end{array}$ & $75 \mathrm{bc}$ & $82 \mathrm{a}$ & $41 \mathrm{~b}$ \\
\hline $\begin{array}{l}\text { Glufosinate }+ \text { fomesafen }+ \text { acetochlor } \mathrm{fb} \\
\text { glufosinate }\end{array}$ & $\begin{array}{l}\text { Early POST fb } \\
\text { late POST }\end{array}$ & $\begin{array}{c}594+263+1,680 \\
594\end{array}$ & $79 a b$ & $83 \mathrm{a}$ & $55 \mathrm{a}$ \\
\hline $\begin{array}{l}\text { Glufosinate + fomesafen + imazethapyr + } \\
\text { acetochlor } \mathrm{fb}\end{array}$ & Early POST fb & $594+263+70+1,680$ & $82 \mathrm{a}$ & $85 \mathrm{a}$ & $58 \mathrm{a}$ \\
\hline glufosinate & late POST & 594 & & & \\
\hline$P$-value & & & 0.002 & $<0.0001$ & $<0.0001$ \\
\hline
\end{tabular}

Common waterhemp densities at 28 DALPOST ranged from 88 to 124 plants $\mathrm{m}^{-2}$ with the POSTonly program (Table 7 ), and ranged from 1 to 78 plants $\mathrm{m}^{-2}$ with PRE fb POST program (Table 6). Monthly precipitation ranging from 28 to $318 \mathrm{~mm}$ in June and July of 2013 and 2014 (Table 1) may have triggered the emergence of common waterhemp; Hartzler et al. (1999) reported that common waterhemp emergence can be enhanced if sufficient moisture is present in the soil. Among PRE fb POST programs, flumioxazin plus cloransulam, chlorimuron plus thifensulfuron plus flumioxazin, alachlor, $S$-metolachlor plus fomesafen or metribuzin, sulfentrazone plus metribuzin, and saflufenacil alone or with dimethenamid applied PRE fb glufosinate applied POST, was associated with the lowest density of common waterhemp $\left(\leq 16\right.$ plants $\mathrm{m}^{-2}$ ), and in most cases provided $\geq 90 \%$ reduction in common waterhemp biomass at 28 DALPOST (Table 6). Legleiter et al. (2009) reported common waterhemp density as low as 2 plants $\mathrm{m}^{-2}$ at 42 DAPOST with a PRE fb POST program, compared with 66 to 76 plants $\mathrm{m}^{-2}$ with a POST-only program. Averaged across treatments at 28 DALPOST, a POST-only program resulted in a common waterhemp density of 99 plants $\mathrm{m}^{-2}$ and a $53 \%$ reduction in waterhemp biomass, while a PRE $\mathrm{fb}$ POST program resulted in 23 plants $\mathrm{m}^{-2}$ and an $86 \%$ reduction in common waterhemp biomass. In an integrated management approach to resistant common waterhemp in Missouri, Schultz et al. (2015) reported $>98 \%$ density reduction using a PRE $\mathrm{fb}$ POST herbicide programs across all row spacings, whereas the two-pass POST program provided $87 \%, 80 \%$, and $50 \%$ density reduction in 19-, 38-, and 76-cm soybean row spacings, respectively.

Soybean Yield. The lowest soybean yield was obtained from the non-treated control $\left(826 \mathrm{~kg} \mathrm{ha}^{-1}\right)$, 
Table 6. Effect of PRE followed by POST herbicide programs on glyphosate-resistant common waterhemp density and biomass reduction, and glufosinate-resistant soybean yield, in field experiments conducted near Fremont, Nebraska in 2013 and 2014. ${ }^{\text {a,b }}$

\begin{tabular}{|c|c|c|c|c|c|c|}
\hline \multirow[b]{2}{*}{ Herbicide program } & \multirow[b]{2}{*}{ Application timing } & \multirow[b]{2}{*}{ Rate } & \multicolumn{2}{|c|}{$\begin{array}{c}\text { Common waterhemp } \\
\text { density }^{c}\end{array}$} & \multirow[b]{2}{*}{ Biomass reduction $^{\mathrm{c}, \mathrm{d}}$} & \multirow[b]{2}{*}{ Soybean yield } \\
\hline & & & 14 DAPRE & 28 DALPOST & & \\
\hline & & $\mathrm{g}$ ae or ai ha ${ }^{-1}$ & _Plan & ats $\mathrm{m}^{-2}$ & - $\%$ & $-\mathrm{kg} \mathrm{ha}^{-1}$ \\
\hline $\begin{array}{l}\text { Flumioxazin } \mathrm{fb} \\
\text { glufosinate }\end{array}$ & $\begin{array}{l}\text { PRE fb } \\
\text { late POST }\end{array}$ & $\begin{array}{l}107 \\
594\end{array}$ & $20 \mathrm{~b}$ & $64 \mathrm{~b}$ & $71 \mathrm{~cd}$ & 1,477 e \\
\hline $\begin{array}{l}\text { Flumioxazin + cloransulam fb } \\
\text { glufosinate }\end{array}$ & $\begin{array}{l}\text { PRE fb } \\
\text { late POST }\end{array}$ & $\begin{array}{c}107+35.3 \\
594\end{array}$ & $1 \mathrm{c}$ & $7 \mathrm{~cd}$ & $92 \mathrm{ab}$ & $1,805 \mathrm{bcd}$ \\
\hline $\begin{array}{l}\text { Chlorimuron + thifensulfuron + } \\
\text { flumioxazin } \mathrm{fb}\end{array}$ & PRE fb & 153 & $0 \mathrm{c}$ & $1 \mathrm{~d}$ & $97 \mathrm{ab}$ & $2,109 \mathrm{a}$ \\
\hline glufosinate & $\begin{array}{l}\text { late POST } \\
\text { PRF fb }\end{array}$ & 594 & & & & \\
\hline $\begin{array}{l}\text { Alachlor fb } \\
\text { glufosinate }\end{array}$ & $\begin{array}{l}\text { PRE fb } \\
\text { late POST }\end{array}$ & $\begin{array}{c}3,360 \\
594\end{array}$ & $1 \mathrm{c}$ & $13 \mathrm{~cd}$ & $90 \mathrm{ab}$ & 1,733 cde \\
\hline $\begin{array}{l}S \text {-metolachlor } \mathrm{fb} \\
\text { glufosinate }\end{array}$ & $\begin{array}{l}\text { PRE fb } \\
\text { late POST }\end{array}$ & $\begin{array}{l}1,420 \\
594\end{array}$ & $34 \mathrm{a}$ & $78 \mathrm{a}$ & $63 \mathrm{~d}$ & 1,480 e \\
\hline $\begin{array}{l}S \text {-metolachlor + imazethapyr fb } \\
\text { glufosinate }\end{array}$ & $\begin{array}{l}\text { PRE fb } \\
\text { late POST }\end{array}$ & $\begin{array}{l}1,420+70 \\
594\end{array}$ & $31 \mathrm{a}$ & $66 \mathrm{ab}$ & $70 \mathrm{~cd}$ & $1,522 \mathrm{de}$ \\
\hline $\begin{array}{l}S \text {-metolachlor }+ \text { fomesafen } \mathrm{fb} \\
\text { glufosinate }\end{array}$ & $\begin{array}{l}\text { PRE fb } \\
\text { late POST }\end{array}$ & $\begin{array}{l}1,480 \\
594\end{array}$ & $0 \mathrm{c}$ & $2 \mathrm{~d}$ & $97 \mathrm{ab}$ & $2,065 \mathrm{ab}$ \\
\hline $\begin{array}{l}S \text {-metolachlor + metribuzin } \mathrm{fb} \\
\text { glufosinate }\end{array}$ & $\begin{array}{l}\text { PRE fb } \\
\text { late POST }\end{array}$ & $\begin{array}{l}2,050 \\
594\end{array}$ & $1 \mathrm{c}$ & $2 \mathrm{~d}$ & $94 \mathrm{ab}$ & $1,984 \mathrm{abc}$ \\
\hline $\begin{array}{l}\text { Sulfentrazone + metribuzin } \mathrm{fb} \\
\text { glufosinate }\end{array}$ & $\begin{array}{l}\text { PRE fb } \\
\text { late POST }\end{array}$ & $\begin{array}{l}570 \\
594\end{array}$ & $1 \mathrm{c}$ & $4 \mathrm{~cd}$ & $92 \mathrm{ab}$ & $1,810 \mathrm{bcd}$ \\
\hline $\begin{array}{l}\text { Saflufenacil fb } \\
\text { glufosinate }\end{array}$ & $\begin{array}{l}\text { PRE fb } \\
\text { late POST }\end{array}$ & $\begin{array}{c}25 \\
594\end{array}$ & $2 c$ & $16 \mathrm{c}$ & 86 bc & $1,633 \mathrm{de}$ \\
\hline $\begin{array}{l}\text { Saflufenacil + dimethenamid fb } \\
\text { glufosinate }\end{array}$ & $\begin{array}{l}\text { PRE fb } \\
\text { late POST }\end{array}$ & $\begin{array}{l}25+950 \\
594\end{array}$ & $0 \mathrm{c}$ & $1 \mathrm{~d}$ & 99 a & $2,210 \mathrm{a}$ \\
\hline$P$-value & & & $<0.0001$ & $<0.0001$ & 0.002 & $<0.0001$ \\
\hline
\end{tabular}

and was comparable with a one-pass POST program $\left(975 \mathrm{~kg} \mathrm{ha}^{-1}\right)$ (Table 7$)$. It was clear that a one-pass POST program of glufosinate plus fomesafen plus imazethapyr plus acetochlor was insufficient to provide effective control due to the continuous emergence pattern of common waterhemp (Table 7). Averaged across treatments, two-pass POST programs provided $1,190 \mathrm{~kg} \mathrm{ha}$ soybean yield compared with $1,803 \mathrm{~kg} \mathrm{ha}^{-}$with a PRE fb POST program (Table 3). Chlorimuron plus thifensulfuron plus flumioxazin, $S$-metolachlor plus fomesafen or metribuzin, or saflufenacil plus dimethenamid, applied PRE fb glufosinate applied POST provided 1,984 to $2,210 \mathrm{~kg} \mathrm{ha}^{-1}$ soybean yield, the highest yields of all the treatments tested (Table 6). Bell et al. (2015) also reported that the use of PRE herbicides improved soybean yield and economic returns compared with POST-only programs for control of Palmer amaranth in GFR soybean. Johnson et al. (2012) further reported that a PRE fb POST program reduced the chance of crop yield loss due to weed interference because of the program's ability to control early- as well as late-emerging weeds. No significant soybean injury was observed in any herbicide program (data not shown), indicating that all programs evaluated in this study were safe for GFR soybean if applied as per the label directions. 
Table 7. Effect of POST-only herbicide programs on glyphosate-resistant common waterhemp density and biomass reduction and glufosinate-resistant soybean yield in field experiments conducted near Fremont, NE in 2013 and 2014.,

\begin{tabular}{|c|c|c|c|c|c|}
\hline \multirow[b]{2}{*}{ Herbicide program } & \multirow[b]{2}{*}{ Application timing } & \multirow[b]{2}{*}{ Rate } & Density $^{c}$ & \multirow[b]{2}{*}{ Biomass reduction ${ }^{\mathrm{c}, \mathrm{d}}$} & \multirow[b]{2}{*}{ Soybean yield } \\
\hline & & & $\overline{28 \text { DALPOST }}$ & & \\
\hline & & $\mathrm{g}$ ae or ai ha ${ }^{-1}$ & Plants $\mathrm{m}^{-2}$ & $\%$ & $\mathrm{~kg} \mathrm{ha}^{-1}$ \\
\hline Non-treated control & - & - & $186 \mathrm{a}$ & - & $826 \mathrm{c}$ \\
\hline $\begin{array}{l}\text { Glufosinate }+ \text { fomesafen }+ \\
\text { imazethapyr + acetochlor }\end{array}$ & Early POST & $594+263+70+1,680$ & $124 \mathrm{~b}$ & $25 \mathrm{~b}$ & 975 bc \\
\hline $\begin{array}{l}\text { Glufosinate } \mathrm{fb} \\
\text { glufosinate }\end{array}$ & $\begin{array}{l}\text { Early POST fb } \\
\text { late POST }\end{array}$ & $\begin{array}{l}594 \\
594\end{array}$ & $107 \mathrm{bc}$ & $49 \mathrm{ab}$ & $1,136 \mathrm{ab}$ \\
\hline $\begin{array}{l}\text { Glufosinate + acetochlor } \mathrm{fb} \\
\text { glufosinate }\end{array}$ & $\begin{array}{l}\text { Early POST fb } \\
\text { late POST }\end{array}$ & $\begin{array}{l}594+1,680 \\
594\end{array}$ & $99 \mathrm{c}$ & $62 \mathrm{a}$ & $1,173 \mathrm{ab}$ \\
\hline $\begin{array}{l}\text { Glufosinate + acetochlor + imazethapyr fb } \\
\text { glufosinate }\end{array}$ & $\begin{array}{l}\text { Early POST fb } \\
\text { late POST }\end{array}$ & $\begin{array}{l}594+1,680+70 \\
594\end{array}$ & $87 \mathrm{c}$ & $61 \mathrm{a}$ & $1,267 \mathrm{a}$ \\
\hline $\begin{array}{l}\text { Glufosinate }+ \text { fomesafen } \mathrm{fb} \\
\text { glufosinate }\end{array}$ & $\begin{array}{l}\text { Early POST fb } \\
\text { late POST }\end{array}$ & $\begin{array}{l}594+263 \\
594\end{array}$ & $95 \mathrm{c}$ & $50 \mathrm{ab}$ & $1,185 \mathrm{a}$ \\
\hline $\begin{array}{l}\text { Glufosinate }+ \text { fomesafen }+ \text { acetochlor } \mathrm{fb} \\
\text { glufosinate }\end{array}$ & $\begin{array}{l}\text { Early POST fb } \\
\text { late POST }\end{array}$ & $\begin{array}{c}594+263+1,680 \\
594\end{array}$ & $88 \mathrm{c}$ & $65 a$ & $1,322 \mathrm{a}$ \\
\hline $\begin{array}{l}\text { Glufosinate + fomesafen + imazethapyr + } \\
\text { acetochlor } \mathrm{fb}\end{array}$ & Early POST fb & $594+263+70+1,680$ & $95 \mathrm{c}$ & $65 a$ & $1,275 \mathrm{a}$ \\
\hline $\begin{array}{l}\text { glufosinate } \\
P \text {-value }\end{array}$ & late POST & 594 & $<0.0001$ & 0.04 & $<0.0001$ \\
\hline
\end{tabular}

${ }^{a}$ Year-by-treatment interactions for glyphosate-resistant common waterhemp density and biomass reduction and soybean yield were not significant; therefore, data were combined across the two years.

${ }^{b}$ Abbreviations: DALPOST, $\mathrm{d}$ after late-POST herbicide application; DAPRE, $\mathrm{d}$ after PRE herbicide application; fb, followed by.

${ }^{\mathrm{c}}$ Means presented within each column with no common letter(s) are significantly different according to Fisher's protected LSD test at $P \leq 0.05$.

${ }^{\mathrm{d}}$ Percent biomass reduction data were arcsine square root transformed before analysis; however, back-transformed original mean values are presented with the interpretation from the transformed data. 
Practical Implications. The evolution of common waterhemp biotypes resistant to glyphosate and ALSinhibitors, and their widespread occurrence in the Midwest, has resulted in a decrease in the number of effective POST herbicide options in GR soybean. Averaged across treatments, glufosinate-based one- or two-pass herbicide programs provided $\leq 50 \%$ control of GR common waterhemp at soybean harvest, while the PRE fb POST programs evaluated in this study provided $82 \%$ control (Table 3). Glufosinate should not be applied after the bloom stage in GFR soybean (Anonymous 2016), and the results of this study revealed that residual herbicides with multiple modes of action applied at soybean planting are a foundation of GR common waterhemp control. The results also suggest that a follow-up application of glufosinate can provide season-long control in GFR soybean. Additionally, using PRE herbicide combinations with multiple modes of action at soybean planting can effectively control Amaranthus, reducing the number of weeds exposed to POST herbicides and thus reducing the effects of selection pressure while improving the efficacy of POST herbicide(s) applied later in the season. Although not evaluated in this study, Aulakh and Jhala (2015) reported that a residual herbicide such as acetochlor or pyroxasulfone can be tank-mixed with a POST glufosinate application for residual control of common waterhemp later in the season.

Herbicide programs in GFR soybean should not rely solely on glufosinate, because repeated applications of glufosinate in the same field may result in the evolution of glufosinate-resistant weeds. For instance, glufosinate-resistant Italian ryegrass (Lolium perenne L. ssp. multiflorum) in California (Avia-Garcia et al. 2012) and goosegrass [Eleusine indica (L.) Gaertn.] in Malaysia (Jalaludin et al. 2010) have been reported. Herbicide programs in GFR soybean have shown effective control of GR giant ragweed (Kaur et al. 2014), common waterhemp (Schultz et al. 2015), Palmer amaranth (Butts et al. 2016; Bell et al. 2015; 2016), johnsongrass [Sorghum halepense (L.) Pers.] (Johnson et al. 2014), and volunteer corn (Chahal and Jhala 2015). The results of this research indicate that there are herbicide programs capable of providing effective control of GR common waterhemp in GFR soybean that can be incorporated into existing cropping systems. Furthermore, multiple-herbicide-resistant soybean cultivars have been developed and tested, and will be available in the marketplace in the near future (Craigmyle et al. 2013a, 2013b; Spaunhorst et al. 2014). These crops can provide an additional tool for controlling the increasing numbers of GR weeds, including common waterhemp (Chahal et al. 2015; Meyer et al. 2015); however, more research is needed on herbicide programs that provide multiple effective modes of action with the judicious use of herbicide-resistant crop technology and other methods for integrated broad-spectrum weed control in order to achieve optimum crop yields in corn and soybean rotations.

\section{Acknowledgments}

The authors would like to thank Jordan Moody, Luke Baldridge, and Ethann Barnes for their help in this project, and Ian Rogers for editing the manuscript.

\section{Literature Cited}

Anderson DD, Roeth FW, Martin AR (1996) Occurrence and control of triazine-resistant common waterhemp (Amaranthus rudis) in field corn (Zea mays). Weed Technol 10:570-575

Anonymous (2016) Liberty® 280 herbicide product label. Research Triangle Park, NC: Bayer Crop Science. $28 \mathrm{p}$

Aulakh JS, Jhala AJ (2015) Comparison of glufosinate-based herbicide programs for broad-spectrum weed control in glufosinate-resistant soybean. Weed Technol 29:419-430

Avia-Garcia WV, Sanchez-Olguin E, Hulting AG, Mallory Smith C (2012) Target site mutation associated with glufosinate-resistance in Italian ryegrass (Lolium perenne L. ssp. multiflorum). Pest Manag Sci 68:1248-1254

Barnett KA, Culpepper SA, York AC, Steckel LE (2013) Palmer amaranth control by glufosinate plus flometuron applied postemergence to WideStrikeß cotton. Weed Technol 27:291-297

Beckie HJ (2006) Herbicide-resistant weeds: management tactics and practices. Weed Technol 20:793-814

Bell HD, Norsworthy JK, Scott RC (2016) Integrating cereals and deep tillage with herbicide programs in glyphosate and glufosinate-resistant soybean for glyphosateresistant Palmer amaranth management. Weed Technol 30:85-98

Bell HD, Norsworthy JK, Scott RC, Popp M (2015) Effect of raw spacing, seeding rate, and herbicide program in glufosinate-resistant soybean on Palmer amaranth management. Weed Technol 29: 390-404

Bell MS, Hager AG, Tranel PJ (2013) Multiple resistance to herbicides from four site-of-action groups in waterhemp (Amaranthus tuberculatus). Weed Sci 61:460-468

Bensch CN, Horak MJ, Peterson D (2003) Interference of redroot pigweed (Amaranthus retroflexus), Palmer amaranth (A. palmeri), and common waterhemp (A. rudis) in soybean. Weed Sci 51:37-43 
Bernards ML, Crespo RJ, Kruger GR, Gaussoin R, Tranel PJ (2012) A waterhemp (Amaranthus tuberculatus) population resistant to 2,4-D. Weed Sci 60:379-384

Butts TR, Norsworthy JK, Kruger GR, Sandell LD, Young BG, Steckel LE, Loux MM, Bradley KW, Conley SP, Stoltenberg DE, Arriaga FJ, Davis VM (2016) Management of pigweeds (Amaranthus spp.) in glufosinate-resistant soybean in the Midwest and Mid-South. Weed Technol 30:355-365

Chahal PS, Aulakh JS, Rosenbaum K, Jhala AJ (2015) Growth stage affects dose response of selected glyphosate-resistant weeds to premix of 2,4-D choline and glyphosate (Enlist Duo ${ }^{\mathrm{TM}}$ herbicide). J Agric Sci 7:1-10

Chahal PS, Jhala AJ (2015) Herbicide programs for control of glyphosate-resistant volunteer corn in glufosinate-resistant soybean. Weed Technol 29:431-443

Chahal GS, Johnson WG (2012) Influence of glyphosate or glufosinate combinations with growth regulator herbicides and other agrochemicals in controlling glyphosate-resistant weeds. Weed Technol 26:638-643

Coetzer E, Al-khatib K., Peterson DE (2002) Glufosinate efficacy on Amaranthus species in glufosinate-resistant soybean. Weed Technol 16:326-331

Craigmyle BD, Ellis JM, Bradley KW (2013a) Influence of herbicide programs on weed management in soybean with resistant to glufosinate and 2,4-D. Weed Technol 27:78-84

Craigmyle BD, Ellis JM, Bradley KW (2013b) Influence of weed height and glufosinate and 2,4-D combinations on weed control in soybean with resistance to 2,4-D. Weed Technol 27:271-280

Culpepper AS (2006) Glyphosate-induced weed shifts. Weed Technol 20:277-281

Hager AG, Wax LM, Stoller EW, Bollero GA (2002a) Common waterhemp interference in soybean. Weed Sci 50:607-610

Hager AG, Wax LM, Bollero GA, Simmons FW (2002b) Common waterhemp (Amaranthus rudis Sauer) management with soil-applied herbicides in soybean (Glycine max (L.) Merr.). Crop Prot 21:277-283

Hartzler RG, Buhler DD, Stoltenberg DE (1999) Emergence characteristics of four annual weed species. Weed Sci 47:578-584

Hausman NE, Singh S, Tranel PJ, Riechers DE, Kaundun SS, Polge ND, Thomas DA, Hager AG (2011) Resistance to HPPD-inhibiting herbicides in a population of waterhemp (Amaranthus tuberculatus) from Illinois, United States. Pest Manag Sci 67:258-261

Heap I (2016a) International Survey of Herbicide Resistant Weeds, Weeds Resistant to EPSP Synthase Inhibitors. http://weedscience. org/summary/moa.aspx?MOAID=12. Accessed June 12, 2016

Heap I (2016b) International Survey of Herbicide Resistant Weeds, Herbicide Resistant Tall Waterhemp Globally. http://weedscience. org/summary/species.aspx? WeedID=219. Accessed June 12, 2016

Horak MJ, Loughin TM (2000) Growth analysis of four Amaranthus species. Weed Sci 48:347-355

Horak MJ, Peterson DE (1995) Biotypes of Palmer amaranth (Amaranthus palmeri) and common waterhemp (Amaranthus rudis) are resistant to imazethapyr and thifensulfuron. Weed Technol 9:192-195

Jalaludin A, Ngim J, Bali BB, Zazali A (2010) Preliminary findings of potentially resistant goosegrass (Eleusine indica) to glufosinate ammonium in Malaysia. Weed Biol Manag 10:256-260
Jhala AJ (2016) Herbicide-resistant weeds. Pages 18-19 in Knezevic SZ, Jhala AJ, Klein RN, Kruger GR, Reicher ZJ, Wilson RG, Shea PJ \& Ogg CL eds, Guide for Weed, Disease, and Insect Management in Nebraska. Lincoln, NE: University of Nebraska-Lincoln Extension

Jhala AJ, Malik MS, Willis JB (2015) Weed control and crop tolerance of micro-encapsulated acetochlor applied sequentially in glyphosate-resistant soybean. Can J Plant Sci 95:973-981

Johnson DB, Norsworthy JK, Scott RC (2014) Herbicide programs for controlling glyphosate-resistant johnsongrass (Sorghum halepense) in glufosinate-resistant soybean. Weed Technol 28:10-18

Johnson G, Breitenbach F, Behnken L, Miller R, Hoverstad T, Gunsolus J (2012) Comparison of herbicide tactics to minimize species shifts and selection pressure in glyphosate-resistant soybean. Weed Technol 26:189-194

Kaur S, Sandell LD, Lindquist JL, Jhala AJ (2014) Glyphosateresistant giant ragweed (Ambrosia trifida) control in glufosinateresistant soybean. Weed Technol 28:569-577

Krausz RF, Young BG (2003) Sulfentrazone enhances weed control of glyphosate in glyphosate-resistant soybean (Glycine max). Weed Technol 17:249-255

Legleiter TR, Bradley KW (2008) Glyphosate and multiple herbicide resistance in common waterhemp (Amaranthus rudis) populations from Missouri. Weed Sci 56:582-587

Legleiter TR, Bradley KW, Massey RE (2009) Glyphosate-resistant waterhemp (Amaranthus rudis) control and economic returns with herbicide programs in soybean. Weed Technol 23:54-61

Logusch EW, Walker DM, McDonald JF, Franz JE (1991) Inhibition of plant glutamine synthetases by substituted phosphinothricins. Plant Plysiol 95:1057-1062

Meyer CJ, Norsworthy JK, Young BG, Steckel LE, Bradley KW, Johnson WG, Loux MM, Davis VM, Kruger GR, Bararpour MT, Ikley JT, Spaunhorst DJ, Butts TR (2015) Herbicide program approaches for managing glyphosateresistant Palmer amaranth and waterhemp in future soybean trait technologies. Weed Technol 29:716-729

[NOAA] National Ocean and Atmospheric Administration (2015) NOWData-NOAA Online Weather Data. http://w2.weather. gov/climate/xmacis.php?wfo=oax. Accessed July 15, 2015

Norsworthy JK, Ward SM, Shaw DR, Llewellyn RS, Nichols RL, Webster TM, Bradley KW, Frisvold G, Powles SB, Burgos NR, Witt WW, Barrett M (2012) Reducing the risks of herbicide resistance: best management practices and recommendations. Weed Sci 60:31-62

Owen MDK (2008) Weed species shifts in glyphosateresistant crops. Pest Manag Sci 64:377-387

Refsell DE, Hartzler RG (2009) Effect of tillage on common waterhemp (Amaranthus rudis) emergence and vertical distribution of seed in the soil. Weed Technol 23:129-133

Riar DS, Norsworthy JK, Steckel LE, Stephenson DO 4th, Eubank TW, Scott RC (2013) Assessment of weed management needs in midsouth United States soybean: a consultant's perspective. Weed Technol 27:612-622

Rosenbaum KK, Bradley KW (2013) A survey of glyphosateresistant waterhemp (Amaranthus rudis) in Missouri soybean fields and prediction of glyphosate resistance in future waterhemp populations based on in-field observations and management practices. Weed Technol 27:656-663 
Sarangi D (2016) Glyphosate-Resistant Common Waterhemp in Nebraska: Biology, Gene Flow, and Management. Ph.D. thesis. Lincoln, NE: University of Nebraska-Lincoln

Sarangi D, Irmak S, Lindquist JL, Knezevic SZ, Jhala AJ (2016) Effect of water stress on the growth and fecundity of common waterhemp (Amaranthus rudis). Weed Sci 64:42-52

Sarangi D, Sandell LD, Knezevic SZ, Aulakh JS, Lindquist JL, Irmak S, Jhala AJ (2015) Confirmation and control of glyphosate-resistant common waterhemp (Amaranthus rudis) in Nebraska. Weed Technol 29:82-92

Schultz JL, Myers BD, Bradley KW (2015) Influence of soybean seeding rate, row spacing, and herbicide programs on the control of resistant waterhemp in glufosinate-resistant soybean. Weed Technol 29:169-176

Sellers BA, Smeda RJ, Johnson WG, Kending JA, Ellersieck MR (2003) Comparative growth of six Amaranthus species in Missouri. Weed Sci 51:329-333

Shoup DE, Al-Khatib K, Peterson DE (2003) Common waterhemp (Amaranthus rudis) resistance to protoporphyrinogen oxidase-inhibiting herbicides. Weed Sci 51:145-150

Spaunhorst DJ, Siefert-Higgins S, Bradley KW (2014) Glyphosateresistant giant ragweed (Ambrosia trifida) and waterhemp (Amaranthus rudis) management in dicamba-resistant soybean (Glycine max). Weed Technol 28:131-141

Steckel GJ, Wax LM, Simmons FW, Phillips WH II (1997) Glufosinate efficacy on annual weeds is influenced by rate and growth stage. Weed Technol 11:484-488

Steckel LE, Sprague CL (2004) Common waterhemp (Amaranthus rudis) interference in corn. Weed Sci 52:359-364

Steckel LE, Sprague CL, Hager AG, Simmons FW, Bollero GA (2003) Effects of shading on common waterhemp (Amaranthus rudis) growth and development. Weed Sci 51:898-903
VanGessel MJ (2001) Glyphosate-resistant horseweed from Delaware. Weed Sci 49:703-705

Waselkov KE, Olsen KM (2014) Population genetics and origin of the native North American agricultural weed waterhemp (Amaranthus tuberculatus; Amaranthaceae). Am J Bot 101:1726-1736

Wiesbrook ML, Johnson WG, Hart SE, Bradley PR, Wax LM (2001) Comparison of weed management systems in narrowrow, glyphosate- and glufosinate-resistant soybean (Glycine max). Weed Technol 15:122-128

Wild A, Manderscheid R (1984) The effect of phosphinothricin on the assimilation of ammonia in plants. Z Naturforsch 39:500-504

Wortman SE (2014) Integrating weed and vegetable crop management with multifunctional air-propelled abrasive grits. Weed Technol 28:243-252

Wu C, Owen MDK (2014) When is the best time to emerge: reproductive phenology and success of natural common waterhemp (Amaranthus rudis) cohorts in the Midwest United States? Weed Sci 62:107-117

Wu C, Owen MDK (2015) When is the best time to emerge-II: Seed mass, maturation, and after ripening of common waterhemp (Amaranthus tuberculatus) natural cohorts. Weed Sci 63:846-854

Young B (2006) Changes in herbicide use patterns and production practices resulting from glyphosate-resistant crops. Weed Technol 20:301-307

Received July 28, 2016, and approved October 24, 2016.

Associate Editor for this paper: Lawrence E. Steckel, University of Tennessee. 